\title{
c-Maf: a bad influence in the education of macrophages
}

\author{
Jose R. Conejo-Garcia and Paulo C. Rodriguez
}

Department of Immunology, H. Lee Moffitt Cancer Center and Research Institute, Tampa, Florida, USA.

\begin{abstract}
Tumor-associated macrophages (TAMs) represent the most abundant hematopoietic cell type in the solid tumor microenvironment. TAMs drive T cell inhibition, promote angiogenesis, and produce tumor growth factors. Although they can paradoxically exert antitumor activity and prime protective immunity, the pathways driving this phenotype remain unclear. In this issue of the $J \mathrm{Cl}$, Liu and colleagues identified the c-Maf transcription factor as a master regulator of protumoral TAM polarization. The authors found that c-Maf promoted TAMs' immunosuppressive activity, governed their metabolic programming, and drove expression of the macrophage differentiation protein, CSF1R. Further, inhibiting c-Maf in myeloid progenitors, and consequent myeloid-lineage cells, including TAMs, delayed tumor growth. Importantly, $\beta$-glucan treatment reduced c-MAF expression in macrophages and monocytes from patients with non-small cell lung cancer (NSCLC) where c-MAF is overexpressed. These results reveal mechanisms whereby myeloid cells drive human cancer progression by thwarting protective immunity and could lead to immunotherapy for most solid malignancies.
\end{abstract}

\section{c-Maf upregulation drives} M2 attributes and enhances immunosuppressive activity

Virtually all solid tumors produce inflammatory factors that pathologically mobilize myeloid cells. At advanced stages of malignant progression, immature cells of both the monocytic and the granulocytic lineage expand from the bone marrow with an immunosuppressive phenotype to accumulate at lymphatic locations and in peripheral blood $(1,2)$. These cells, termed myeloidderived suppressor cells (MDSCs), can also home to tumor beds (3-5), where MDSCs of the monocytic lineage (or M-MDSCs) mature into even more immunosuppressive $\mathrm{MHC}-\mathrm{II}^{+}$macrophages (6-8). Although most tumor-associated macrophages (TAMs) originate from M-MDSCs (1), proliferating tissue-resident macrophages can also substantially contribute to the TAM pool in some tumors (9). Independently of their origin, most TAMs are associated with tumor-promoting and immunosuppressive activities. Based on the capacity of macrophages to undergo polarization programs that endow them with different functional activities, the phenotype of TAMs is associated with M2-like features (10).

The M1/M2 categorization stems from classic in vitro studies identifying M1 macrophages as proinflammatory, microbicidal cells, whereas the M2 phenotype was associated with antiinflammatory properties, including the secretion of immunosuppressive cytokines. Although this binary categorization is complicated by a continuum of differentiation and heterogeneous polarization programs in the tumor microenvironment (TME), most TAMs clearly exhibit M2-like attributes, including the amplified expression of the mannose recep-

Related Article: p. 2081

Conflict of interest: JRCG declares research support, intellectual property income, and stock options from Anixa Biosciences and Compass Therapeutics.

Copyright: (c) 2020, American Society for Clinical Investigation.

Reference information: / Clin Invest. 2020;130(4):1629-1631. https://doi.org/10.1172/JCI135444.

tor CD206 and other scavenger receptors, arginase-1, indoleamine 2,3-dioxygenase (IDO), and tumor-promoting cytokines, including IL-10, IL-6, vascular endothelial growth factor A (VEGF-A), and TGF- $\beta$ (9, 11), while showing inhibited expression of M1 mediators such as IL-12. However, the molecular drivers of this M2-like phenotype, or how to reprogram macrophages into antitumor effectors, remain incompletely understood.

In this issue of the JCI, Liu and colleagues reported that the leucine zipper transcription factor, c-Maf, is heavily overexpressed in M2-polarized macrophages. Silencing of c-Maf in M2 cells, or ectopic expression in M1 macrophages, reversed or promoted M2-like polarizing attributes, respectively, including IL-10 and arginase I expression. Accordingly, macrophages from the fetal liver of c-Maf-KO mice failed to polarize into M2-like cells, and showed reduced immunosuppressive activity, compared with their c-Maf-competent counterparts (12).

To gain mechanistic insight into how c-Maf regulates M2-like macrophage polarization, Liu et al. performed ChIP-sequencing experiments to identify stretches of DNA that bound c-Maf. Many intergenic regions, along with direct binding to a conserved sequence located $3 \mathrm{~kb}$ downstream of the Csflr transcription start site, were identified (12). The Csflr gene encodes the receptor for colony-stimulating factor 1 (CSF1), a cytokine that controls the production, differentiation, and function of macrophages (13) and MDSCs (14, 15), for which targeting approaches are currently in clinical development (16). The functional relevance of c-Maf binding to the Csfir locus was confirmed using luciferase reporters in M2-polarization experiments. Accordingly, inhibiting c-Maf with a mycotoxin, Nivalenol, resulted in Csf1r (also known as CD115) downregulation concomitantly with decreased TAM immunosuppressive activity (12), suggesting that c-Maf drives an immunosuppressive phenotype in macrophages by regulating Csf1r expression (Figure 1). 


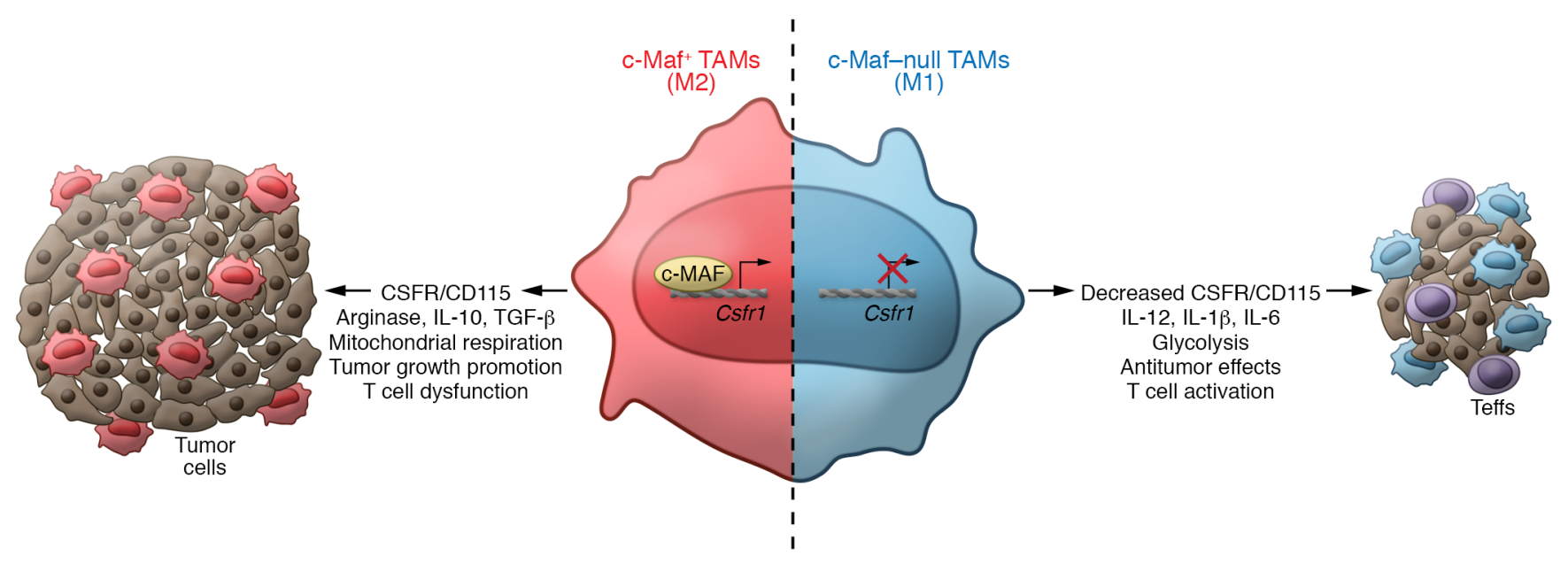

Figure 1. c-Maf promotes M2 polarization in TAMs. In NSCLC, macrophages upregulate the expression of c-Maf, which transcriptionally regulates the expression of $\mathrm{Csfr} 1$ and promotes functional and metabolic polarization to M2-like phenotypes and drives T cell suppression. Liu et al. describe that inhibition or elimination of c-Maf in macrophages reprograms their metabolism and function to M1-like cells that promote antitumor responses.

\section{c-Maf promotes M2-like polarization of macrophages in cancer}

Because metabolic activity alters the functional properties of macrophages, Liu et al. went on to demonstrate that inhibition of c-Maf impaired mitochondrial activity and increased baseline glycolytic flux, a phenotype observed in M1 macrophages. Complementary studies using stable isotope-resolved metabolomics (SIRM) linked to mass spectrometry revealed that c-Maf positively regulates TCA cycle activity along with N-glycosylation (12). Collectively, these results point to a role for c-Maf as the driver of a metabolic switch in M2-polarized macrophages.

As expected, c-Maf was significantly overexpressed in (M2-like) macrophages infiltrating transplantable murine tumors, in which c-Maf silencing again reduced their suppressive activity and Csf1r expression, and caused metabolic reprogramming. Interestingly, bone marrowderived MDSCs lacked c-Maf expression, and c-Maf deficiency failed to change the magnitude of MDSC mobilization. Furthermore, turning on c-Maf did not accelerate the conversion of M-MDSCs into $\mathrm{MHC}-\mathrm{II}^{+}$macrophages in vitro, suggesting that c-Maf drives M2-like polarization in plastic $\mathrm{MHC}-\mathrm{II}^{+}$macrophages once they have matured at tumor beds, and not in their MDSC precursors. However, c-Maf expression was also found in circulating $\mathrm{CD} 14^{+} \mathrm{CD} 68^{-}$precursors in cancer patients (12). Hence, the pos- sibility that c-Maf expression drives the conversion of M-MDSCs/monocytes into M2-like macrophages at tumor beds, in conjunction with hypoxia (8), CSF (7), or exosomes released from tumor-educated mesenchymal stem cells (6) cannot be ruled out. Further studies evaluating the crosstalk of c-Maf in different populations of myeloid cells in tumors would be relevant, as the expression of the c-Mafmodulated inhibitory factors Csfr1, IL-10, arginase I, and IDO has been associated with MDSC-mediated regulation (14, 15, 17). Also, the mechanisms driving the expression and activity of c-Maf in TAMs remain unknown.

\section{c-Maf ablation in myeloid cells delays malignant progression}

Because constitutive ablation of c-Maf is embryonically lethal, Liu et al. generated a conditional c-Maf $\mathrm{KO}$, to confirm the role of c-Maf in driving tumor-promoting activities in myeloid cells. As expected, the absence of c-Maf in myeloid cells resulted in delayed tumor growth, compared with mice bearing c-Maf-competent myeloid cells (Figure 1). Of note, c-Maf ablation using the conditional system selectively reduced the accumulation of tumor-promoting polymorphonuclear MDSCs (PMN-MDSCs) at tumor beds, which are also major suppressors of antitumor immunity. Nevertheless, c-Maf-deficient TAMs also exhibited reduced suppressive effects on effector $\mathrm{T}$ cells, along with decreased expression of CD206 and increased MHC-II levels (12). Accordingly, increased infiltration of IFN- $\gamma$-producing $\mathrm{T}$ cells in tumors from mice without c-Maf expression in myeloid cells could be attributed, at least in part, to phenotypic changes in TAMs, although the effects of c-Maf in granulocytes (including PMN-MDSCs) remain unclear. It also remains to be elucidated whether the M1 switch and the metabolic transformation occurring in c-Maf-deficient TAMs allows them to prime protective antitumor $\mathrm{T}$ cell immunity, or perhaps the M1-like phenotype provides the ability to directly elicit direct antitumor cytotoxicity. Strategies to reprogram immunosuppressive myelopoiesis in tumors could thereby boost the effects of immunotherapy in the clinic.

One of the most appealing aspects of the study by Liu and colleagues is the effectiveness of c-Maf inhibitors. Combined anti-PD-1 and c-Maf inhibition, but not any individual intervention, caused significant delays in the progression of poorly immunogenic Lewis lung carcinoma in mice, associated with tumorinfiltrating lymphocytes with superior production of IFN- $\gamma$. Therefore, although none of the c-Maf inhibitors used are truly specific, the work of Liu et al. points to a unique target to expand the range of patients responsive to immune checkpoint inhibitors. Furthermore, because the immunosuppressive effects conferred by c-Maf overexpression in M2-like macrophages appear to be dependent on Csf1r regulation, the current study indirectly 
supports combinatorial interventions using existing antibodies or small molecules targeting CSF1R to overcome resistance to PD-1 blockers (12).

\section{Natural compounds can inhibit c-Maf in cancer patients}

Liu et al. support the translational potential of their observations by exploring c-Maf expression in tumor- and blood-associated cells from patients with non-small cell lung cancer (NSCLC). Compared with healthy donors, c-Maf was overexpressed in tissue-associated macrophages with M2-like $\left(\mathrm{CD}_{163^{+}}\right)$features as well as in circulating immature myeloid cells/monocytes. In an effort to identify clinically applicable drugs targeting c-Maf, the authors investigated the effects of fungal $\beta$-glucans on macrophage polarization. Using yeast-derived particulate $\beta$-glucans, Liu et al. transformed bone marrow- or tumor-derived M2-like macrophages into an M1-like phenotype that corresponded with downregulated c-Maf levels. Accordingly, $\beta$-glucans delayed tumor growth through a mechanism that is dependent on macrophages, as macrophage depletion decreased $\beta$-glucan therapeutic efficacy. Because $\beta$-glucans had similar effects on reducing c-MAF expression in human TAMs, a clinical trial was initiated in NSCLC patients. Although no information on outcome is yet available, a notable reduction in c-Maf mRNA expression was identified in circulating CD $14^{\text {dim }}{ }^{\text {CD } 16^{+}}$myeloid cells, in association with increased TNF- $\alpha$ and decreased IL-10 levels (12). $\beta$-Glucan could therefore provide a clinical alternative to CSF1R targeting to decrease the immunosuppressive burden elicited by myeloid cells.
Given the magnitude of the immunosuppressive burden dependent on myeloid cells in solid tumors, it seems unlikely that simply rescuing $\mathrm{T}$ cells from intrinsic checkpoint inhibitory pathways will ever allow effective control of most advanced tumors. The results of Liu and colleagues (12) pave the way for further investigations into how to target myeloid cells to unleash the power of antitumor immunity.

\section{Acknowledgments}

This work was supported by NIH grants R01CA157664, R01CA124515, R01CA178687, and R01CA211913 (to JRCG), and R01CA184185 and R01CA233512 (to PCR).

Address correspondence to: Jose R. Conejo-Garcia, H. Lee Moffitt Cancer Center and Research Institute, 12902 Magnolia Drive, Tampa, Florida 33612, USA. Phone: 813.745.8282; Email: jose. conejo-garcia@moffitt.org.

1. Kumar V, Patel S, Tcyganov E, Gabrilovich DI. The nature of myeloid-derived suppressor cells in the tumor microenvironment. Trends Immunol. 2016;37(3):208-220.

2. Svoronos N, et al. Tumor cell-independent estrogen signaling drives disease progression through mobilization of myeloid-derived suppressor cells. Cancer Discov. 2017;7(1):72-85.

3. Umansky V, Blattner C, Gebhardt C, Utikal J. The role of myeloid-derived suppressor cells (MDSC) in cancer progression. Vaccines (Basel). 2016;4(4):E36

4. Gabrilovich DI, Ostrand-Rosenberg S, Bronte V. Coordinated regulation of myeloid cells by tumours. Nat Rev Immunol. 2012;12(4):253-268.

5. Bronte V, et al. Recommendations for myeloid-derived suppressor cell nomenclature and characterization standards. Nat Commun. 2016;7:12150.
6. Biswas S, et al. Exosomes produced by mesenchymal stem cells drive differentiation of myeloid cells into immunosuppressive M2-polarized macrophages in breast cancer. J Immunol. 2019;203(12):3447-3460.

7. Ugel S, De Sanctis F, Mandruzzato S, Bronte V. Tumor-induced myeloid deviation: when myeloid-derived suppressor cells meet tumor-associated macrophages. J Clin Invest. 2015;125(9):3365-3376.

8. Corzo CA, et al. HIF-1 $\alpha$ regulates function and differentiation of myeloid-derived suppressor cells in the tumor microenvironment. JExp Med 2010;207(11):2439-2453.

9. Laviron M, Boissonnas A. Ontogeny of tumor-associated macrophages. Front Immunol. 2019;10:1799.

10. Locati M, Curtale G, Mantovani A. Diversity, mechanisms, and significance of macrophage plasticity. Annu Rev Pathol. 2020;15:123-147.

11. Sica A, Schioppa T, Mantovani A, Allavena P. Tumour-associated macrophages are a distinct M2 polarised population promoting tumour progression: potential targets of anti-cancer therapy. Eur J Cancer. 2006;42(6):717-727.

12. Liu M, et al. Transcription factor c-Maf is a checkpoint that programs macrophages in lung cancer. JClin Invest. 2020;130(4):2081-2096.

13. Ries $\mathrm{CH}$, et al. Targeting tumor-associated macrophages with anti-CSF-1R antibody reveals a strategy for cancer therapy. Cancer Cell. 2014;25(6):846-859.

14. Huang B, et al. Gr- $1^{+} \mathrm{CD} 115^{+}$immature myeloid suppressor cells mediate the development of tumor-induced $\mathrm{T}$ regulatory cells and $\mathrm{T}$-cell anergy in tumor-bearing host. Cancer Res. 2006;66(2):1123-1131

15. Youn JI, Nagaraj S, Collazo M, Gabrilovich DI. Subsets of myeloid-derived suppressor cells in tumor-bearing mice. J Immunol. 2008;181(8):5791-5802.

16. Cannarile MA, Weisser M, Jacob W, Jegg AM, Ries $\mathrm{CH}$, Rüttinger D. Colony-stimulating factor 1 receptor (CSF1R) inhibitors in cancer therapy. J Immunother Cancer. 2017;5(1):53.

17. Rodriguez PC, et al. Arginase I-producing myeloid-derived suppressor cells in renal cell carcinoma are a subpopulation of activated granulocytes. Cancer Res. 2009;69(4):1553-1560. 